\title{
Essential Self-Adjointness and Invariance of the Essential Spectrum for Dirac Operators
}

\author{
By \\ Masaharu ARA* and Osanobu YAMADA**
}

\section{$\S 1$. Introduction}

In the present paper we are concerned with the Dirac operator

$$
L=\sum_{j=1}^{3} \alpha_{j} D_{j}+\beta+Q(x) \quad\left(x \in \mathbb{R}^{3}, D_{j}=-i \frac{\partial}{\partial x_{j}}\right),
$$

where $\alpha_{j}(j=1,2,3)$ and $\alpha_{4}=\beta$ are Hermitian symmetric, constant, $4 \times 4$ matrices and satisfy the anti-commutation relations

$$
\alpha_{j} \alpha_{k}+\alpha_{k} \alpha_{j}=2 \delta_{j k} I \quad(j, k=1,2,3,4)
$$

( $I$ is the $4 \times 4$ unit matrix). Throughout this paper the potential $Q(x)$ is assumed to be an Hermitian symmetric $4 \times 4$ matrix-valued measurable function. The Dirac operator is treated in the Hilbert space $\mathscr{L}^{2}=\left[L^{2}\left(\mathbb{B}^{3}\right)\right]^{4}$ associated with the norm

$$
\|f\|_{\lambda^{2}}=\sqrt{\int_{R^{3}}}|f(x)|^{2} d x<\infty
$$

and the inner product

$$
(f, g)=\int_{\boldsymbol{R}^{3}}\langle f(x), g(x)\rangle d x
$$

for $f(x)={ }^{t}\left(f_{1}(x), f_{2}(x), f_{3}(x), f_{4}(x)\right)$ and $g(x)={ }^{t}\left(g_{1}(x), g_{2}(x), g_{3}(x), g_{4}(x)\right)$, where $|f(x)|^{2}=\sum_{j=1}^{4}\left|f_{j}(x)\right|^{2}$ and $\langle f(x), g(x)\rangle=\sum_{j=1}^{4} f_{j}(x) \overline{g_{j}(x)}$.

Let $H$ be the restriction of $L$ to $\mathscr{C}_{0}^{\infty} \equiv\left[C_{0}^{\infty}\left(\mathbb{R}^{3}\right)\right]^{4}$ and $H_{0}$ be $H$ with $Q \equiv 0$, where $C_{0}^{\infty}\left(\mathbb{R}^{3}\right)$ is the totality of infinitely differentiable functions with compact support in $\mathbb{R}^{3}$. It is obvious that $H$ and $H_{0}$ are symmetric in $\mathscr{L}^{2}$ if $|Q(x)|$ $\in L_{2,10 c}$. (For any matrix $A$, we denote by $|A|$ the squere root of the greatest

Communicated by S. Matsuura, May 18, 1981.

* Faculty of Economics, Ritsumeikan University, Kyoto 603, Japan.

*** Faculty of Science and Engineering, Ritsumeikan University, Kyoto 603, Japan. 
eigenvalue of $A^{*} A$.) It is well known that $H_{0}$ is essentially self-adjoint. The essential self-adjointness of $H$ (especially, with a scalar potential) has been studied extensively by many authors (see e.g. Kalf-Schmincke-Walter-Wüst [6] and its references). Let us sketch some known results in the case of the Coulomb potential

$$
Q(x)=(e / r) I\left(e: \text { real constant } ; r=\sqrt{x_{1}^{2}+x_{2}^{2}+x_{3}^{2}}\right) .
$$

Kato [7; Chap. V, Theorem 2.10] shows that $H$ is essentially self-adjoint if $|e| \leqq 1 / 2$, and

$$
D(\bar{H})=D\left(\overline{H_{0}}\right)
$$

if $|e|<1 / 2$. This is proved by Kato-Rellich-Wüst perturbation theorem and the well-known inequality

$$
\begin{aligned}
\int_{\boldsymbol{R}^{3}} \frac{|u(x)|^{2}}{r^{2}} d x & \leqq 4 \int_{\boldsymbol{R}^{3}} \sum_{j=1}^{3}\left|\frac{\partial u}{\partial x_{j}}\right|^{2} d x \\
& =4 \int_{\boldsymbol{R}^{3}}\left|\sum_{j=1}^{3} \alpha_{j} \frac{\partial u}{\partial x_{j}}\right|^{2} d x
\end{aligned}
$$

for $u \in \mathscr{C}_{0}^{\infty}$, so that this can be extended immediately for $H$ with a matrix-valued potential $Q(x)$ as follows: if

$$
|x||Q(x)| \leqq 1 / 2
$$

$H$ is essentially self-adjoint, and if

$$
\sup _{x}|x||Q(x)|<1 / 2,
$$

(1.3) holds.

Let us return to the Coulomb potential (1.2). Rellich [9] and Weidmann [12] show that $H$ is essentially self-adjoint if and only if $|e| \leqq \sqrt{3} / 2$. One may expect that $H$ with a matrix-valued potential $Q(x)$ satisfying

$$
|x||Q(x)| \leqq \sqrt{3} / 2
$$

is essentially self-adjoint. But this expectation is not correct. Indeed, Arai [1] shows that for any $\varepsilon>0$ there exists an Hermitian symmetric potential $Q_{\varepsilon}(x)$ satisfying

$$
|x|\left|Q_{\varepsilon}(x)\right|=1 / 2+\varepsilon
$$

for which the Dirac operator $H$ is not essentially self-adjoint. (This result is re-proved in Arai [14].)

Our aim in this note is to show a sufficient condition for the essential self- 
adjointness of the Dirac operator with matrix-valued potentials (Theorem 2.1 (1)), and one for (1.3) (Theorem $2.1(2)$ ). We shall also prove the invariance of the essential spectrum (Theorem 2.2). When we apply Theorem 2.1 (2) to the Coulomb potential (1.2), we have that $H$ is essentially self-adjoint and (1.3) holds if $|e|<\sqrt{3} / 2$ (see Example 6.1). We can also show that (1.3) holds even if the equality holds in (1.5) under some additional condition (Example 6.2).

In Section 2, we shall state the assumptions and Theorems 2.1 and 2.2. The former will be proved in Section 3 and the latter in Section 5. Section 4 is devoted to prove a lemma used in the proof of Theorem 2.1. In Section 6, we shall give applications and a remark to Theorem 2.1 .

\section{§2. Assumptions and Results}

The following condition on the potential $Q(x)$ is assumed in this note:

(A.I) $Q(x)$ can be decomposed as $Q(x)=Q_{1}(x)+Q_{2}(x)$ such that $Q_{1}(x)$ and $Q_{2}(x)$ are Hermitian symmetric $4 \times 4$ matrix-valued measurable functions satisfying the following (A.II) and (A.III), respectively.

(A.II) There exist positive constants $\rho$ and $e<\sqrt{3} / 2$ such that

$$
Q_{1}(x)^{2}+\frac{i}{2 r}\left\{\alpha_{r} Q_{1}(x)-Q_{1}(x) \alpha_{r}\right\} \leqq \frac{e^{2}}{r^{2}} I
$$

in $B_{\rho}$, and $Q_{1}(x) \equiv 0$ in $B_{\rho}^{c}$ (the complement of $B_{\rho}$ ), where $B_{\rho}=\left\{x \in \mathbb{R}^{3}|| x \mid<\rho\right\}$ and $\alpha_{r}=\sum_{j=1}^{3} \frac{x_{j}}{r} \alpha_{j}(A \leqq B$ means that $B-A$ is a positive semi-definite matrix $)$.

(A.III) For any $\varepsilon>0$ and $R>0$ there exists a positive constant $C(\varepsilon, R)$ such that

$$
\left\|Q_{2} u\right\|_{\mathscr{L}^{2}\left(B_{R}\right)} \leqq \varepsilon \sum_{j=1}^{3}\left\|\frac{\partial u}{\partial x_{j}}\right\|_{\mathscr{L}^{2}}+C(\varepsilon, R)\|u\|_{\mathscr{L}^{2}}
$$

for any $u \in \mathscr{C}_{0}^{\infty}$.

Under the condition (A) we have

Theorem 2.1. (1) The Dirac operator $H$ defined as

$$
H u=L u, \quad u \in D(H) \equiv \mathscr{C}_{0}^{\infty}
$$

is essentially' self-adjoint.

(2) Assume moreover that $C(\varepsilon, R)$ in the condition (A.III) can be chosen independent of $R>0$. Then (1.3) holds.

It is well known that the essential spectrum $\sigma_{\text {ess }}\left(\bar{H}_{0}\right)$ of $\bar{H}_{0}$ is equal to $\boldsymbol{R} \backslash(-1,+1)$. 
Theorem 2.2. Assume that

$$
\left|Q_{2}(x)\right| \longrightarrow 0 \text { as }|x| \longrightarrow \infty
$$

in addition to the condition $(A)$. Then we have

$$
\sigma_{\text {ess }}(\bar{H})=\sigma_{\text {ess }}\left(\bar{H}_{0}\right)=\boldsymbol{R} \mid(-1,+1) .
$$

Remark 2.3. The assumption (A.II) and

$$
\alpha_{r}^{2}=I
$$

which is a direct consequence of the anti-commutation relation (1.1), imply that there exists a positive constant $C_{1}$ such that

$$
r\left|Q_{1}(x)\right| \leqq C_{1} .
$$

Remark 2.4. The following potentials $Q_{2}(x)$, for example, satisfy the condition (A.III). We can take $C(\varepsilon, R)$ in (A.III) as independent of $R>0$, if $Q_{2}(x)$ satisfies the following condition without underlined parts.

(III-1) Each component of $Q_{2}(x)$ is a locally $L^{3}$-function (Gross [4]).

(III-2) The function of $x \in \mathbb{R}^{3}$

$$
\int_{|x-y| \leqq 1} \frac{\left|Q_{2}(y)\right|^{2}}{|x-y|^{1+\delta}} d y
$$

is locally bounded for some $\delta>0$ (Evans [3]).

(III-3) The function

$$
\int_{|x-y| \leqq \delta} \frac{\left|Q_{2}(y)\right|^{2}}{|x-y|} d y
$$

is locally bounded and tends to zero as $\delta \downarrow 0$ uniformly on every compact set (Schechter [10]).

Remark 2.5. Let $0<\rho^{\prime}<\rho$ and $\chi_{\rho^{\prime}}(x)$ be the characteristic function of $B_{\rho^{\prime}}$. Replace $Q_{1}(x)$ and $Q_{2}(x)$ by $Q_{1}(x) \equiv \chi_{\rho^{\prime}}(x) Q_{1}(x)$ and $\tilde{Q}_{2}(x) \equiv$ $\left(1-\chi_{\rho^{\prime}}(x)\right) Q_{1}(x)+Q_{2}(x)$. Then $\left(1-\chi_{\rho}(x)\right) Q_{1}(x)$ is bounded by virtue of $(2.2)$, so that $\widetilde{Q}_{2}(x)$ satisfies (A.III) with $Q_{2}$ replaced by $\widetilde{Q}_{2}$. It is obvious that $\widetilde{Q}_{1}(x)$ satisfies (A.II) with $Q_{1}$ and $\rho$ replaced by $\widetilde{Q}_{1}$ and $\rho^{\prime}$. Thus we may assume $\rho$ in (A.II) to be sufficiently small.

Remark 2.6. The number $\sqrt{3} / 2$ in (A.II) is best possible, as will be shown in Remark 6.3. 


\section{§3. Proof of Theorem 2.1}

Before the proof of Theorem 2.1 we will give some preparations. In consequence of (1.1) we obtain

$$
\left(\sum_{j=1}^{3} \xi_{j} \alpha_{j}+\beta\right)^{2}=\left(|\xi|^{2}+1\right) I
$$

for $\xi \in \mathbb{R}^{3}$. It follows from (3.1) that $D\left(H_{0}\right)=\mathscr{H}^{1}$ and

$$
\|u\|_{\mathscr{R}^{1}}=\left\|\bar{H}_{0} u\right\|_{\mathscr{L}^{2}}
$$

for every $u \in \mathscr{H}^{1}$. Here the Sobolev space $\mathscr{H}^{1}$ cosists of all the $\mathscr{L}^{2}$-functions with the first derivatives in $\mathscr{L}^{2}$, normed by

$$
\|f\|_{\mathscr{C}^{1}}=\sqrt{\int_{\mathbb{R}^{3}}\left\{|f(x)|^{2}+\sum_{j=1}^{3}\left|\frac{\partial f}{\partial x_{j}}\right|^{2}\right\} d x} .
$$

From these considerations we have

Lemma 3.1. The inverse $\bar{H}_{0}^{-1}$ exists and bounded.

The following Lemma will be an important tool in our proof.

Lemma 3.2. Let $T$ be a densely defined closable linear operator in a Hilbert space $X$ and $A$ a linear operator such that $D(T) \subset D(A)$. Suppose that there exists a positive constant $k<1$ such that

$$
\|A u\| \leqq k\|T u\|
$$

for every $u \in D(T)$ and that the closure $\bar{T}$ has a bounded inverse on $X$. Then we have

(1) $T+A$ is closable.

(2) $D(\overline{T+A})=D(\bar{T})$.

(3) $\overline{T+A}$ has a bounded inverse on $X$.

(For the proof, see Kato [7; p. 190, p. 196].)

Lemma 3.3. Let $Q(x)$ be an Hermitian symmetric potential whose components are of the class $L_{\text {loc }}^{2}$ and $\chi_{R}(x)$ the characteristic function of $B_{R}$. Assume that $H_{R}=H_{0}+\chi_{R} Q$ is essentially self-adjoint for any $R>0$. Then $H=H_{0}+Q$ is essentially self-adjoint.

(For the proof, see Chernoff [2].)

Proof of Theorem 2.1. We shall first see the essentiall self-adjointness of 
$H_{1}=H_{0}+Q_{1}$. The idea of the proof, which is due to Schmincke [11] is to decompose $H_{1}$ to the sum

$$
H_{1}=\left(H_{0}+\frac{i k}{2 r} \alpha_{r}\right)+\left(-\frac{i k}{2 r} \alpha_{r}+Q_{1}\right)
$$

for $0<k<1$ and apply Lemma 3.2 twice to $T=H_{0}, A=\frac{i k}{2 r} \alpha_{r}$ and

$$
T=H_{0}+\frac{i k}{2 r} \alpha_{r}, \quad A=-\frac{i k}{2 r} \alpha_{r}+Q_{1} .
$$

It follows from (1.4), (2.1) and (3.2) that

$$
\left\|\frac{i k}{2 r} \alpha_{r} u\right\|_{\mathscr{L}^{2}} \leqq k\left\|\sum_{j=1}^{3} \alpha_{j} D_{j} u\right\|_{\mathscr{L}^{2}} \leqq k\left\|H_{0} u\right\|_{\mathscr{L}^{2}}
$$

for $u \in \mathscr{C}_{0}^{\infty}$. Therefore Lemma 3.2 is applicable to $T=H_{0}$ and $A=\frac{i k}{2 r} \alpha_{r}$ for every $k$ such that $0<k<1$ in view of Lemma 3.1. Thus we obtain that $\overline{H_{0}+\frac{i k}{2 r} \alpha_{r}}$ has the bounded inverse on $\mathscr{L}^{2}$ and $D\left(\overline{\left.H_{0}+\frac{i k}{2 r} \alpha_{r}\right)}=D\left(\bar{H}_{o}\right)\right.$.

Under the assumption (A.II) we have

Lemma 3.4. If $\rho>0$ is sufficiently small, we can find a positive number $k<1$ such that

$$
\left\|\left(-\frac{i k}{2 r} \alpha_{r}+Q_{1}\right) u\right\|_{\mathscr{L}^{2}} \leqq \sqrt{k}\left\|_{i}\left(H_{0}+\frac{i k}{2 r} \alpha_{r}\right) u\right\|_{\mathscr{L}^{2}}
$$

for any $u \in \mathscr{C}_{0}^{\infty}$.

The above Lemma 3.4 will be proven in Section 4.

Since we may assume $\rho>0$ to be sufficiently small (Remark 2.5), we are permitted to make use of Lemma 3.4 for our potential $Q_{1}(x)$. Then Lemma 3.2 implies that $\lambda=0$ belongs to the resolvent set of $\bar{H}_{1}$ and $D\left(\bar{H}_{1}\right)=D\left(\bar{H}_{0}\right)$. As the resolvent set of $\bar{H}_{1}$ is open, the deficiency index of $\bar{H}_{1}$ is $(0,0)$ and, consequently, $\bar{H}_{1}$ is self-adjoint, which yields the essential self-adjointness of $H_{1}$.

Since $\bar{H}_{0} \bar{H}_{1}^{-1}$ is bounded on $\mathscr{L}^{2}$ from the closed graph theorem, the condition (A.III) gives us that

$$
\begin{aligned}
\left\|\chi_{R} Q_{2} u\right\|_{\mathscr{L}^{2}} & \leqq \varepsilon\left\|H_{0} u\right\|_{\mathscr{L}^{2}}+C(\varepsilon, R)\|u\|_{\mathscr{L}^{2}} \\
& \leqq \varepsilon\left\|\bar{H}_{0} \bar{H}_{1}^{-1}\right\| \cdot\left\|H_{1} u\right\|_{\mathscr{L}^{2}}+C(\varepsilon, R)\|u\|_{\mathscr{L}^{2}}
\end{aligned}
$$

for every $\varepsilon>0, R>0$ and $u \in \mathscr{C}_{0}^{\infty}$. If we take $\varepsilon>0$ so small that $\varepsilon\left\|\bar{H}_{0} \bar{H}_{1}^{-1}\right\|<1$, we have from (3.4) that

$$
H_{0}+\chi_{R} Q=H_{1}+\chi_{R} Q_{2} \quad(R>\rho)
$$


is essentially self-adjoint by virtue of Kato [7; p. 288]. Therefore Lemma 3.2 assures the essential self-adjointness of $H=H_{0}+Q$. Thus we complete the proof of (1).

Let us prove (2) of Theorem 2.1. By virtue of (3.4), we have

$$
\left\|\chi_{R} Q_{2} u\right\|_{\mathscr{L}^{2}} \leqq \varepsilon\left\|H_{1} u\right\|_{\mathscr{L}^{2}}+C(\varepsilon)\|u\|_{\mathscr{L}^{2}}
$$

for every $\varepsilon>0, R>0$ and $u \in \mathscr{C}_{0}^{\infty}$, where we may assume that $C(\varepsilon)$ is independent of $R>0$ from our assumption on (2). Taking the limit as $R \rightarrow \infty$ in (3.5), we have

$$
\left\|Q_{2} u\right\|_{\mathscr{L}^{2}} \leqq \varepsilon\left\|H_{1} u\right\|_{\mathscr{L}^{2}}+C(\varepsilon)\|u\|_{\mathscr{L}^{2}},
$$

for every $\varepsilon>0$ and $u \in \mathscr{C}_{0}^{\infty}$, which gives by means of Kato $[7 ;$ p. 288] that $D(\bar{H})=D\left(\bar{H}_{1}\right)=D\left(\bar{H}_{0}\right)$.

\section{§4. Proof of Lemma 3.4}

Definition 4.1. We shall use the same notations as given in Schmincke [11]:

$$
\begin{aligned}
p_{r} & =-i\left(\frac{\partial}{\partial r}+\frac{1}{r}\right), \\
\vec{L} & =\left(L_{1}, L_{2}, L_{3}\right) \\
& =\left(\frac{x_{2}}{i} \frac{\partial}{\partial x_{3}}-\frac{x_{3}}{i} \frac{\partial}{\partial x_{2}}, \frac{x_{3}}{i} \frac{\partial}{\partial x_{1}}-\frac{x_{1}}{i} \frac{\partial}{\partial x_{3}}, \frac{x_{1}}{i} \frac{\partial}{\partial x_{2}}-\frac{x_{2}}{i} \frac{\partial}{\partial x_{1}}\right), \\
\vec{\sigma} & =\left(\sigma_{1}, \sigma_{2}, \sigma_{3}\right)=-i\left(\alpha_{2} \alpha_{3}, \alpha_{3} \alpha_{1}, \alpha_{1} \alpha_{2}\right), \\
\vec{L}^{2} & =L_{1}^{2}+L_{2}^{2}+L_{3}^{2}, \\
\vec{\sigma} \cdot \vec{L} & =\sigma_{1} L_{1}+\sigma_{2} L_{2}+\sigma_{3} L_{3} .
\end{aligned}
$$

The following Lemma plays an important part in the present section.

Lemma 4.2. (1) The following identity holds:

$$
\begin{aligned}
& \left(\sum_{j=1}^{3} \alpha_{j} D_{j}+\beta\right) r^{k}\left(\sum_{j=1}^{3} \alpha_{j} D_{j}+\beta\right) \\
& \quad=k r^{k-2}+p_{r} r^{k} p_{r}+r^{k-2}\left(\vec{L}^{2}+k \vec{\sigma} \cdot \vec{L}\right)-i k r^{k-1} \alpha_{r} \beta+r^{k} .
\end{aligned}
$$

(2) Let $S^{2}=\left\{\omega \in \mathbb{R}^{3}|| \omega \mid=1\right\}$ and $f(\omega)$ be an arbitrary $C^{2}$-function on $S^{2}$. Then we have

$$
\int_{S^{2}}\left\langle\left(\vec{L}^{2}+\vec{\sigma} \cdot \vec{L}\right) f(\omega), f(\omega)\right\rangle d \omega \geqq 0
$$

The first assertion (1), which follows from direct calculations, is a generalization of Wüst [13, Lemma 2], The latter one (2) is due to Schmincke [11, 
Lemma 4]. It is well-known in many books of quantum mechanics that the total angular momentum

$$
\left(\vec{L}+\frac{\vec{\sigma}}{2}\right)^{2} \geqq \frac{3}{4} I
$$

which and $\sigma_{j}^{2}=I(j=1,2,3)$ imply

$$
\vec{L}^{2}+\vec{\sigma} \cdot \vec{L}=\left(\vec{L}+\frac{\vec{\sigma}}{2}\right)^{2}-\frac{3}{4} I \geqq 0 .
$$

Proof of Lemma 3.4. Let $0<k<1$ and $u \in \mathscr{C}_{0}^{\infty}$. Then Lemma 4.2 shows

$$
\begin{aligned}
& \left\|H_{0} u+\frac{i k}{2 r} \alpha_{r} u\right\|_{\mathscr{L}^{2}}^{2}=\left\|r^{\frac{k}{2}}\left(\sum_{j=1}^{3} \alpha_{j} D_{j}+\beta\right)\left(\frac{u}{r^{k / 2}}\right)\right\|_{\mathscr{L}^{2}}^{2} \\
& =\int_{\boldsymbol{R}^{3}}\left\langle\left(\sum_{j=1}^{3} \alpha_{j} D_{j}+\beta\right) r^{k}\left(\sum_{j=1}^{3} \alpha_{j} D_{j}+\beta\right)\left(\frac{u}{r^{k / 2}}\right), \frac{u}{r^{k / 2}}\right\rangle d x \\
& \geqq \int_{\boldsymbol{R}^{3}}\left(\frac{k}{r^{2}}-\frac{k}{r}+1\right)|u|^{2} d x+\int_{\boldsymbol{R}^{3}} r^{k}\left|p_{r}\left(\frac{u}{r^{k / 2}}\right)\right|^{2} d x \\
& \quad+k \int_{\boldsymbol{R}^{3}}\left\langle\frac{1}{r^{2}}\left(\vec{L}^{2}+\vec{\sigma} \cdot \vec{L}\right) u, u\right\rangle d x \\
& \geqq \int_{\boldsymbol{R}^{3}}\left(k-k r+r^{2}\right) \frac{|u|^{2}}{r^{2}} d x,
\end{aligned}
$$

where we have used $\vec{L}^{2} \geqq 0$ and $\left|\alpha_{r} \beta\right| \leqq I$.

The condition (A.II) and (2.2) give

$$
\begin{aligned}
& \int_{\boldsymbol{R}^{3}}\left|-\frac{i k}{2 r} \alpha_{r} u+Q_{1} u\right|^{2} d x \\
& \quad=\int_{\boldsymbol{R}^{3}}\left\langle\left[\frac{k^{2}}{4 r^{2}}+Q_{1}^{2}+\frac{i k}{2 r}\left\{\alpha_{r} Q_{1}-Q_{1} \alpha_{r}\right\}\right] u, u\right\rangle d x \\
& \leqq \int_{\boldsymbol{R}^{3}}\left\{\frac{k^{2}}{4 r^{2}}+\frac{e^{2}}{r^{2}} \chi_{\rho}(x)+\frac{C_{1}(1-k)}{r^{2}} \chi_{\rho}(x)\right\}|u|^{2} d x .
\end{aligned}
$$

Here (4.1) and (4.2) are combined to obtain

$$
\begin{aligned}
& k\left\|H_{0}+\frac{i k}{2 r} \alpha_{r} u\right\|_{\mathscr{L}^{2}}^{2}-\left\|-\frac{i k}{2 r} \alpha_{r} u+Q_{1} u\right\|_{\mathscr{L}^{2}}^{2} \\
& \quad \geqq \int_{\boldsymbol{R}^{3}}\left[\frac{3}{4} k^{2}-k^{2} r+k r^{2}-\left\{e^{2}+C_{1}(1-k)\right\} \chi_{\rho}(x)\right] \frac{|u|^{2}}{r^{2}} d x .
\end{aligned}
$$

In order to see the non-negativity of (4.3) we have only to show

$$
\begin{aligned}
& \frac{3}{4} k^{2}-k^{2} r+k r^{2} \geqq 0 \quad(r \geqq \rho), \\
& \frac{3}{4} k^{2}-k^{2} \rho-e^{2}-C_{1}(1-k) \geqq 0 .
\end{aligned}
$$


The inequality (4.4) holds for every $r>0$, since the discriminant of the quadratic function is $k^{4}-3 k^{3}=k^{3}(k-3)<0$. The later inequality (4.5) follows from $e^{2}<\frac{3}{4}$ in the condition (A.II) and letting $k$ approach to 1 and $\rho$ to 0 . Q.E.D.

\section{§5. Proof of Theorem 2.2}

In this section we shall prove $\sigma_{\text {ess }}(\bar{H})=\sigma_{\text {ess }}\left(\bar{H}_{0}\right)$ under the condition (A) and

$$
\left|Q_{2}(x)\right| \longrightarrow 0 \text { as }|x| \longrightarrow \infty \text {. }
$$

Recall that $\lambda \in \sigma_{\text {ess }}(S)$ for a self-adjoint operator $S$ if and only if there exists an orthonormal sequence $\left\{u_{n}\right\} \subset D(S)$ such that $(S-\lambda) u_{n} \rightarrow 0$ strongly as $n \rightarrow \infty$ (cf., e.g., Kato [7]). Let $\gamma(t)$ be a $C^{\infty}$-function defined on $\mathbb{R}$ such that $0 \leqq \gamma(t)$ $\leqq 1$ in $\mathbb{R}, \gamma(t)=1$ for $t \leqq 0$ and $\gamma(t)=0$ for $t \geqq 1$. We put $\gamma_{R}(x)=\gamma(|x|-R)$ $(R>0)$. Then we have

$$
\left\{\begin{array}{l}
\gamma_{R}(x)=1 \quad \text { for } \quad|x|<R, \\
\gamma_{R}(x)=0 \quad \text { for } \quad|x|>R+1 \\
\sum_{j=1}^{3}\left|\frac{\partial}{\partial x_{j}} \gamma_{R}(x)\right| \leqq C_{2}
\end{array}\right.
$$

with some constant $C_{2}$ not depending on $R>0$. Note here that (1.4) holds for $u \in D\left(\bar{H}_{0}\right)=\mathscr{H}^{1}$. (A.III) and (5.1) imply

$$
\left\|Q_{2} u\right\|_{\mathscr{L}^{2}} \leqq\left\|\bar{H}_{0} u\right\|_{\mathscr{L}^{2}}+C_{3}\|u\|_{\mathscr{L}^{2}},
$$

for any $u \in D\left(H_{0}\right)$ with some positive constant $C_{3}$.

Let $u \in \mathscr{H}^{1}$. Then it follows from (1.4), (2.2), (3.2) and (5.2) that

$$
\begin{aligned}
\|Q u\|_{\mathscr{L}^{2}} \leqq & Q_{1} u\left\|_{\mathscr{L}^{2}}+\right\| Q_{2} \gamma_{R} u\left\|_{\mathscr{L}^{2}}+\right\| Q_{2}\left(1-\gamma_{R}\right) u \|_{\mathscr{L}^{2}} \\
\leqq & C_{1}\left\|\frac{1}{r} \gamma_{R} u\right\|_{\mathscr{L}^{2}}+\left\|\bar{H}_{0}\left(\gamma_{R} u\right)\right\|_{\mathscr{L}^{2}}+C_{3}\left\|\gamma_{R} u\right\|_{\mathscr{L}^{2}}+\left(\sup _{|x| \geqq R}\left|Q_{2}(x)\right|\right)\|u\|_{\mathscr{L}^{2}} \\
\leqq & \left(2 C_{1}+1\right)\left\|\left(\bar{H}_{0}-z\right)\left(\gamma_{R} u\right)\right\|_{\mathscr{L}^{2}}+\left\{\left(2 C_{1}+1\right)|z|+C_{3}\right\}\left\|\gamma_{R} u\right\|_{\mathscr{L}^{2}} \\
& +\left(\sup _{|x| \geqq R}\left|Q_{2}(x)\right|\right)\|u\|_{\mathscr{L}^{2}}
\end{aligned}
$$

for any complex number $z$ and positive number $R>\rho$.

Now let $\lambda$ be in $\sigma_{\text {ess }}\left(\bar{H}_{0}\right)$ and $\left\{u_{n}\right\}$ be an orthonormal sequence in $\mathscr{L}^{2}$ such that $\left(\bar{H}_{0}-\lambda\right) u_{n} \rightarrow 0$ as $n \rightarrow \infty$. Since $\left\{u_{n}\right\}$ are bounded in $\mathscr{H}^{1}$, it follows from Rellich's theorem that there exists a subsequence $\left\{u_{n_{k}}\right\} \subset\left\{u_{n}\right\}$ such that $\left\{u_{n_{k}}\right\}$ tends to 0 locally in $\mathscr{L}^{2}$. Substitute $u=u_{n_{k}}$ and $z=\lambda$ into (5.4) and use (5.2) to obtain 


$$
\varlimsup_{k \rightarrow \infty}\left\|Q u_{n_{k}}\right\|_{\mathscr{L}^{2}} \leqq \sup _{|x| \geqq R}\left|Q_{2}(x)\right|,
$$

which and the assumption (5.1) imply that $Q u_{n_{k}} \rightarrow 0$. Thus we have

$$
(\bar{H}-\lambda) u_{n_{k}}=\left(\bar{H}_{0}-\lambda\right) u_{n_{k}}+Q u_{n_{k}} \longrightarrow 0,
$$

which shows $\lambda \in \sigma_{\text {ess }}(H)$.

Reversely, let $\lambda \in \sigma_{\text {ess }}(\bar{H})$ and $\left\{u_{n}\right\}$ be an orthonormal sequence in $\mathscr{L}^{2}$ such that $(\bar{H}-\lambda) u_{n} \rightarrow 0$. Since $D\left(\bar{H}_{0}\right)=D(\bar{H})$ by virtue of Theorem 2.1(2), we have $u_{n} \in D\left(\bar{H}_{0}\right)$ and that $\bar{H}_{0}(\bar{H}-\lambda+i)^{-1}$ is bounded so that the boundedness of $\left\|u_{n}\right\|_{\mathscr{L}^{2}}+\left\|\bar{H} u_{n}\right\|_{\mathscr{L}^{2}}$ implies the boundedness of $\left\|u_{n}\right\|_{\mathscr{L}^{2}}+\left\|\bar{H}_{0} u_{n}\right\|_{\mathscr{L}^{2}}$. Thus we can choose a subsequence $\left\{u_{n_{k}}\right\} \subset\left\{u_{n}\right\}$ such that $u_{n_{k}} \rightarrow 0$ locally in $\mathscr{L}^{2}$ as above. The boundedness of $\left(\bar{H}_{0}-z\right)(\bar{H}-z)^{-1}$ for $z=\lambda+i$, and the estimate (5.2) and (5.4) yield

$$
\begin{aligned}
& \|Q u\|_{\mathscr{L}^{2}} \leqq\left(2 C_{1}+1\right)\left\{\left\|\left(\bar{H}_{0}-z\right)(\bar{H}-z)^{-1}\right\|\left\|(\bar{H}-\lambda)\left(\gamma_{R} u\right)\right\|_{\mathscr{L}^{2}}+\right. \\
& \left.+\left\|\gamma_{R} u\right\|_{\mathscr{L}^{2}}\right\}+\left\{\left(2 C_{1}+1\right)(|\lambda|+1)+C_{3}\right\}\left\|\gamma_{R} u\right\|_{\mathscr{L}^{2}}+\left(\sup _{|x|>R}\left|Q_{2}(x)\right|\right)\|u\|_{\mathscr{L}^{2}} \\
& \leqq C_{4}\|(\bar{H}-\lambda) u\|_{\mathscr{L}^{2}}+C_{5}\|u\|_{\mathscr{L}^{2}\left(B_{R+1}\right)}+\left(\sup _{|x|>R}\left|Q_{2}(x)\right|\right)\|u\|_{\mathscr{L}^{2}},
\end{aligned}
$$

where we put

$$
\begin{aligned}
& C_{4}=\left(2 C_{1}+1\right)\left\|\left(\bar{H}_{0}-z\right)(\bar{H}-z)^{-1}\right\|, \\
& C_{5}=C_{2} C_{4}+\left(2 C_{1}+1\right)+\left\{\left(2 C_{1}+1\right)(|\lambda|+1)+C_{3}\right\} .
\end{aligned}
$$

Put $u=u_{n_{k}}$ in (5.6). Then we have again (5.5). Thus we have

$$
\left(\bar{H}_{0}-\lambda\right) u_{n_{k}}=(\bar{H}-\lambda) u_{n_{k}}-Q u_{n_{k}} \longrightarrow 0,
$$

which implies $\lambda \in \sigma_{\text {ess }}(\bar{H})$ and completes the proof.

\section{§6. Examples}

Example 6.1. Let $P(x)$ be an Hermitian symmetric $4 \times 4$ matrix-valued measurable function satisfying

$$
|P(x)| \leqq e<\sqrt{3} / 2
$$

and commutes with $\alpha_{r}$. Then $Q(x)=r^{-1} P(x)$ satisfies the assumptions of Theorem 2.1(2). In particular, if $Q(x)$ is a scalar potential $Q(x)=r^{-1} p(x) I$ with $|p(x)| \leqq e<\sqrt{3} / 2$, then $Q(x)$ satisfies this assumption. The latter example includes results of Gustafson and Rejto [5; Theorem 2.1] and Landgren and Rejto [8; Theorem 2.1 and its Corollaries]. 
Example 6.2. Let $Q(x)$ be an Hermitian symmetric matrix-valued measurable function satisfying one of the following conditions:

(1) There exists a constant $e$ such that

$$
r|Q(x)| \leqq e<1 / 2 .
$$

(2) The function $r Q(x)$ is continuous in $\bar{B}_{\rho}$ for some $\rho>0$,

$$
r|Q(x)| \leqq 1 / 2,
$$

and $1 / 2$ is not an eigenvalue of $i r \alpha_{r} Q(x)$ for any $x$ in $\bar{B}_{\rho}$.

Then $Q(x)$ satisfies the assumptions of Theorem 2.1(2).

Proof. The proof under the assumption (1) will be easily seen by mimicking the inequality (6.2) below. So, we only prove this Lemma under the assumption (2). Let $x$ be fixed in $\bar{B}_{\rho}$. For any $\xi \in \boldsymbol{C}^{4}$, we have

$$
\begin{aligned}
& \left\langle\left(r^{2} Q(x)^{2}+\frac{i r}{2}\left\{\alpha_{r} Q(x)-Q(x) \alpha_{r}\right\}\right) \xi, \xi\right\rangle \\
& \quad=|r Q(x) \xi|^{2}-\operatorname{Im}\left\langle r Q(x) \xi, \alpha_{r} \xi\right\rangle \\
& \quad \leqq \frac{1}{4}|\xi|^{2}+\frac{1}{2}|\xi|^{2}=\frac{3}{4}|\xi|^{2},
\end{aligned}
$$

where we used the Schwarz inequality

$$
\begin{aligned}
& -\operatorname{Im}\left\langle r Q(x) \xi, \alpha_{r} \xi\right\rangle \leqq\left|\left\langle r Q(x) \check{\xi}, \alpha_{r} \ddot{\xi}\right\rangle\right| \\
& \quad \leqq|r Q(x) \xi|\left|\alpha_{r} \xi\right|
\end{aligned}
$$

and the assumption (6.1). We claim that the equality sign does not hold in the inequality sign ' $\leqq$ ' in (6.2) for any non-trivial $\zeta$. Indeed, if the equality sign holds for some non-trivial vector $\xi$. Then

$$
|r Q(x) \xi|=(1 / 2)|\xi|
$$

and the both inequality signs ' $\leqq$ ' in (6.3) must be equality signs for this $\zeta$. The latter equality implies

$$
r Q(x) \xi=c \alpha_{r} \xi
$$

with some constant $c$. Then the former equality implies $-\operatorname{Im} c=|c|$ so that $c=-i d, d \geqq 0$, which with (6.4) implies $d=1 / 2$. Thus (6.5) becomes $\operatorname{ir}_{r} Q(x) \xi$ $=(1 / 2) \xi$, which contradict our assumption. Thus we have

$$
\left\langle\left(r^{2} Q(x)+\frac{i r}{2}\left\{\alpha_{r} Q(x)-Q(x) \alpha_{r}\right\}\right) \xi, \xi\right\rangle<(3 / 4)|\xi|^{2} \quad \text { for } \quad \xi \neq 0 .
$$

Since the left hand side of this inequality is a continuous function of $(x, \xi)$ on the 
compact set $\bar{B}_{\rho} \times\left\{\right.$ the unit sphere in $\left.C^{4}\right\}$, we have (2.1) with $e^{2}<3 / 4$, which completes the proof.

Remark 6.3. As in usual textbooks on the quantum mechanics, we put

$$
\begin{aligned}
& \sigma_{1}=\left(\begin{array}{ll}
0 & 1 \\
1 & 0
\end{array}\right), \quad \sigma_{2}=\left(\begin{array}{cc}
0 & -i \\
i & 0
\end{array}\right), \quad \sigma_{3}=\left(\begin{array}{cc}
1 & 0 \\
0 & -1
\end{array}\right), \\
& \sigma_{r}=\sum_{j=1}^{3} \sigma_{j} \cdot x_{j} / r \\
& \alpha_{j}=\left(\begin{array}{cc}
0 & \sigma_{j} \\
\sigma_{j} & 0
\end{array}\right) \quad(j=1,2,3) \quad \text { and } \quad \beta=\left(\begin{array}{cc}
I & 0 \\
0 & -I
\end{array}\right) .
\end{aligned}
$$

The counter example of the first author [1] mentioned in Section 1 is as follow:

Let $a$ and $b$ be real numbers such that

$$
1 / 4>(b-1)^{2}-a^{2} \neq 0 .
$$

Then the operator $H$ with $Q$ defined by

$$
Q(x)=\frac{1}{r}\left(\begin{array}{cc}
a I & i b \sigma_{r} \\
-i b \sigma_{r} & a I
\end{array}\right)
$$

is not essentially self-adjoint.

In this case, we have

$$
\begin{aligned}
V & \equiv r^{2} Q(x)^{2}+(i r / 2)\left(\alpha_{r} Q(x)-Q(x) \alpha_{r}\right) \\
& =\left(\begin{array}{cc}
a^{2}+b^{2} & 2 i a b \sigma_{r} \\
-2 i a b \sigma_{r} & a^{2}+b^{2}
\end{array}\right)+\frac{i}{2}\left[\left(\begin{array}{cc}
-i b & a \sigma_{r} \\
a \sigma_{r} & i b
\end{array}\right)-\left(\begin{array}{cc}
i b & a \sigma_{r} \\
a \sigma_{r} & -i b
\end{array}\right)\right] \\
& =\left(a^{2}+b^{2}\right) I+b V_{1^{\prime}}
\end{aligned}
$$

where we used $\sigma_{r}^{2}=I$ and put $V_{1}=\left(\begin{array}{cc}I & 2 i a \sigma_{r} \\ -2 i a \sigma_{r} & -I\end{array}\right)$.

Since $V_{1}^{2}=\left(1+4 a^{2}\right) I$ and $V_{1}$ is not a scalar (times the unit matrix), the eigenvalues of $V_{1}$ are $\pm \sqrt{1+4 a^{2}}$ so that

$$
|V|=a^{2}+b^{2}+|b| \sqrt{1+4 a^{2}} .
$$

Under the assumption (6.6),

$$
\begin{gathered}
|V|>2 b^{2}-2 b+3 / 4+|b| \sqrt{4 b^{2}-8 b+4} \\
\quad=2 b(b-1)+2|b(b-1)|+3 / 4 \geqq 3 / 4
\end{gathered}
$$

so that the condition (2.1) does not hold. Let $b=1 / 2$ and $0<a<1 / 2$. Then (6.6) is satisfied and $|V|$ tends to $3 / 4$ from above as $a \searrow 0$. Thus the number $\sqrt{3 / 2}$ in the Assumption (A.II) is best posible. 


\section{References}

[ 1 ] Arai, M., On essential self-adjointness of Dirac operators, RIMS Kokyuroku, 242, Kyoto Univ., (1975), 10-21.

[2] Chernoff, P. R., Schrödinger and Dirac operators with singular potentials and hyperbolic equations, Pacific J. Math., 72 (1977), 361-382.

[3] Evans, W. D., On the unique selfadjoint extension of the Dirac operator and the existence of the Green matrix, Proc. London Math. Soc., 20 (1970), 537-557.

[4] Gross, L., The Cauchy problem for the coupled Maxwell and Dirac equations, Comm. Pure Appl. Math., 19 (1966), 1-15.

[5] Gustafson, K. E. and Rejto, P. A., Some essentially self-adjoint Dirac operators with spherically symmetric potentials, Israel J. Math., 14 (1973), 63-75.

[6] Kalf, H., Schmincke, U, -W., Walter, J. and Wüst, R., On the spectral theory of Schrödinger and Dirac operators with strongly singular potentials, Lecture notes in mathematics, Springer, 448 (1974), 182-226.

[ 7] Kato, T., Perturbation Theory for Linear Operators, Springer, 1966.

[8] Landgren, J. J. and Rejto, P. A., An application of the maximum principle to the study of essential self-adjointness of Dirac operators, I, J. Math. Phys., 20 (1979), 22042211.

[9] Rellich, F., Eigenwerttheorie partieller Differentialgleichungen II, vervielfältigtes Vorlesungsmanuskript, Göttingen, 1953.

[10] Schechter, M.. Spectra of Partial Differential Operators, North-Holland, 1971.

[11] Schmincke. U.-W., Essential self-adjointness of Dirac operators with a strongly singular potential, Math. Z., 126 (1972), 71-81.

[12] Weidmann, J., Oszillationsmethoden für Systeme gewöhnlicher Differentialgleichungen, Math. Z., 119 (1971), 349-373.

[13] Wüst, R., Distinguished self-adjoint extensions of Dirac operatos constructed by means of cut-off potentials, Math. Z., 141 (1975), 93-98.

[14] Arai, M., On essential selfadjointness, distinguished selfadjoint extension and essential spectrum of Dirac operators with matrix valued potentials, to appear.

[15] Yamada, O., Notes on the essential self-adjointness of Dirac operators, Memoirs of the Research Institute of Science and Engineering, Ritsurneikan University, 40 (1981), 1-8.

Added in proof. Recently Yamada [15] shows that Theorem 2.1(1) holds even if $e=\sqrt{3} / 2$ in (A.II). 
